\title{
Application of 3D Projection Profilometry in the High Speed Impaction Surface Deformation Measurement Research
}

\author{
Eryi Hu1,2 (D) \\ ${ }^{1}$ School of Mechatronic Engineering, China University of Mining and Technology, Xuzhou, China \\ ${ }_{2}^{2}$ Jiangsu Key Laboratory of Mine Mechanical and Electrical Equipment, China University of Mining and Technology, \\ Xuzhou, China \\ Email: horyhu@126.com
}

How to cite this paper: Hu, E.Y. (2020) Application of 3D Projection Profilometry in the High Speed Impaction Surface Deformation Measurement Research. Journal of Signal and Information Processing, 11, 103-115.

https://doi.org/10.4236/jsip.2020.114006

Received: September 24, 2020

Accepted: November 23, 2020

Published: November 26, 2020

Copyright ( 2020 by author(s) and Scientific Research Publishing Inc. This work is licensed under the Creative Commons Attribution International License (CC BY 4.0).

http://creativecommons.org/licenses/by/4.0/ (c) (i) Open Access

\begin{abstract}
In order to study the strength of the composite material plate problems, need to adopt a nondestructive testing method to obtain the specimen surface under the effect of high-speed impact regularity of shape. The projection profilometry was used to measure the surface profile or the full field deformation. Furtherly, by using the Fourier transform algorithm, there is only one frame of captured image which is needed in the measurement, so that it can be introduced into the high speed impaction procedure measurement. An experimental system, which was contained with an impact setup and the projection profilometry measurement part, was constructed for the impaction action characteristic research. The metallic impact object can be launched by a gas gun or a spin fan, respectively. The detected object is manufactured by composite materials. In order to increase the surface deformation measurement accuracy, the calibration method and the error was discussed with different calibration specimen. And then, the proposed profilometry measurement method is proved by the gas gun and spin fan projectile test. The surface deformation of the manufactured composite plates and fan case are measured in the impaction procedure. So that the impact action details can be described much more clearly than the traditional video monitoring method.
\end{abstract}

\section{Keywords}

Projection Profilometry, Fourier Transform Algorithm, Deformation Measurement, Impaction Monitoring

\section{Introduction}

As the developing of the Charge Coupled Device (CCD) camera and image 
processing algorithm are growing day by day, the 3-D optical and image measuring technology is widely used in the industry inspection [1] [2] [3]. Especially in the surface profile and surface deformation measurement area, it is easy to obtain the full field displacement, so that the deformation and the strain can be obtained by a simply calculation [4] [5]. All of the previous measuring methods can be divided into two types of techniques, active3-D shape measurement and passive3-D shape measurement. In the active3-D shape measurement, a regular pattern [6], such as a well-defined array of lines, is adhered to the measured sample's surface, and an image of this grid is obtained before and after deformation. An automatic analysis of the dynamic deformation of this fine grid has allowed the full-field measurement of in-plane displacement [7]. In the active 3-D shape measurement techniques, the triangulation-based physical principle is much more popular used in the dynamic object measurement. The structured pattern was projected onto the detected object surface, so that it can be demodulated by the surface profile. The images were captured by the CCD or Complementary Metal Oxide Semiconductor (COMS) camera, and then the measured object's information can be extracted by image processing algorithm.

In the projection grating profilometry of a dynamic impaction measurement, the parallel with cosine function modulated intensity is projected onto the detected object. The mainly two kinds of phase extracting methods are phase-shifting measurement profilometry (PMP) and Fourier transformation profilometry (FTP). The PMP is a mature method with high accuracy [8] [9], but this method is generally applied to the profile measurement of a static object. Generally, only one image can be obtained in the dynamic measurement, so the Fourier transform technique is used to extract the phase information [10] [11] [12] [13]. The fundamental frequency component, which includes the phase change information caused by the surface height of the detected object, is filtered from the frequency domain [14] [15] [16]. However, few studies have addressed the issue of the deformation measurement of the composite material specimen by using a high speed single frame CCD camera.

In this paper, the projection profilometry and the Fourier transform method are introduced into the surface deformation inspection of the manufactured composite plates. The experimental system is constructed to research the whole procedure of the impaction deformation. Some interesting experimental results are present to prove the feasibility of the proposed method.

\section{The Principle of the Deformation Measurement}

\subsection{The Principle of Optical Trigonometry}

As shown in Figure 1, the parallel with cosine function modulated intensity is projected onto the reference plane at an incidence angle of $\alpha$. The pitch of the fringe patterns is $p$. A point $Q$ on the object surface would have two corresponding shadow point on the reference plane, in the observation direction (point $R$ ) and the projection direction (point $S$ ). 


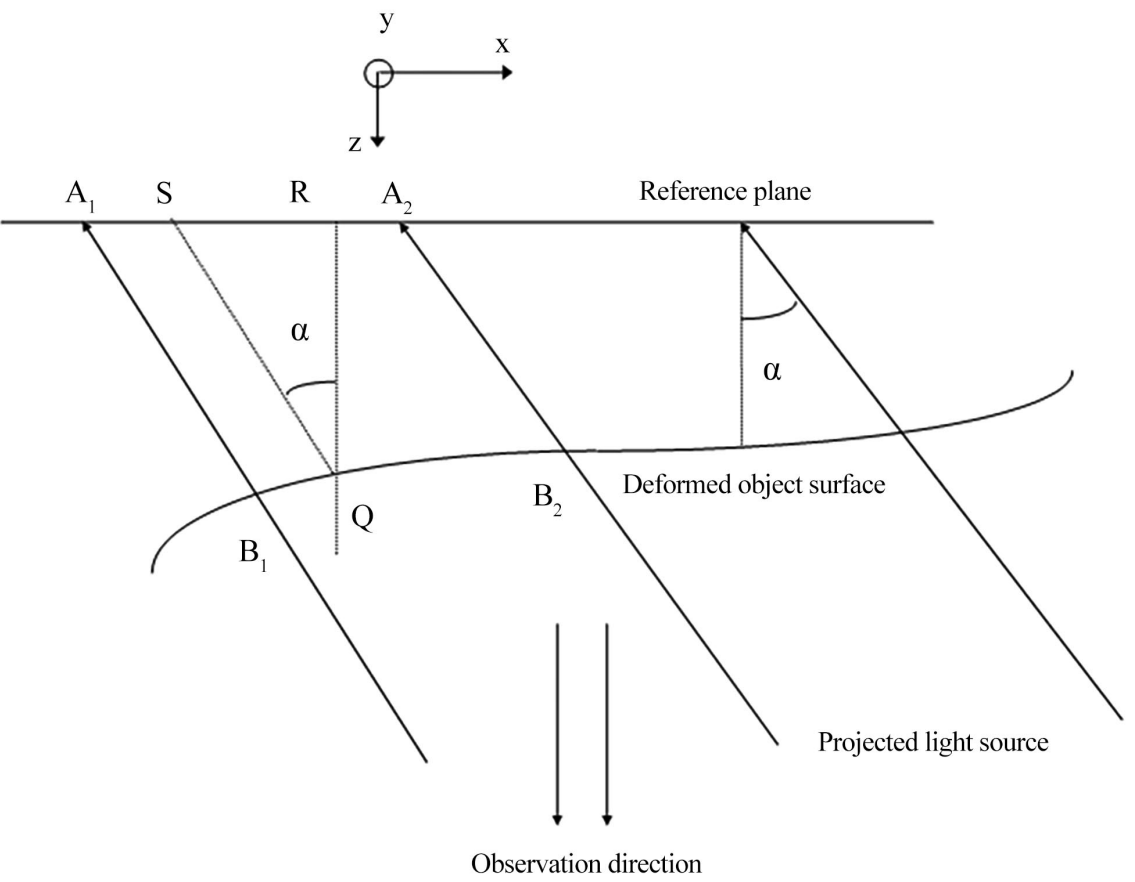

Figure 1. Geometric relationship between projection gratings and surface height.

$$
R S=P\left(\varphi_{S}-\varphi_{R}\right) / 2 \pi
$$

As $Q S$ is parallel to the incidence direction, point $Q$ on the deformed object and point $S$ on the reference surface would have the same phase angle. So

$$
R S=P\left(\varphi_{Q}-\varphi_{R}\right) / 2 \pi
$$

From simple geometric, the surface height $R Q$ is expressed as follow.

$$
R Q=R S / \tan \alpha
$$

Substituting Equation (2) into Equation (3), we have

$$
R Q=\left(\varphi_{Q}-\varphi_{R}\right) \frac{p}{2 \pi \tan \alpha}=\left(\varphi_{Q}-\varphi_{R}\right) k
$$

Thus from Equation (4), the object surface height can be obtained, if the phase difference between the reference and the detected surface gratings can be measured. Furthermore, the coefficient $k$ is defined to indicate the relationship. In order to reduce the system error, the coefficient can be obtained by a calibration test. As only one frame of fringe pattern can be captured by the high-speed CCD camera in every moment of the impact procedure, Fourier transform method is used to extract the phase change.

\subsection{Fourier Transform Phase Extracting Technique}

When the detected object is put on the reference plane, the deformed fringe pattern captured by a CCD camera can be expressed as:

$$
I(x, y)=a(x, y)+b(x, y) \cos \left[2 \pi f_{0} x+\Phi(x, y)\right]
$$

where $a(x, y)$ is the background intensity, $b(x, y)$ is the amplitude of the 
gratings, $f_{0}$ is the spatial frequency, and $\Phi(x, y)$ is the phase change caused by the surface height of the object. The signal $\Phi(x, y)$ is modulated by a constant high-frequency signal $f_{0}$. Thus, Equation (5) can be written as

$$
I(x, y)=a(x, y)+c(x, y) \exp \left(j 2 \pi f_{0} x\right)+c^{*}(x, y) \exp \left(-j 2 \pi f_{0} x\right) .
$$

where $c(x, y)=[b(x, y) / 2] \exp \{j[\Phi(x, y)]\}$, and $c^{*}(x, y)$ is the complex conjugate of $c(x, y)$. The Fourier transform of $I(x, y)$ with respect of $x$ becomes

$$
F[I(x, y)]=A(f, y)+C\left(f-f_{0}, y\right)+C^{*}\left(f+f_{0}, y\right),
$$

where $F[], A()$ and $C\left(\right.$ ) represent the Fourier spectra, and $C^{*}$ is the complex conjugate of $C$.

As the frequency of $a(x, y), b(x, y)$ and $\Phi(x, y)$ are much lower than $f_{0}$, the function $C\left(f-f_{0}, y\right)$ can be filtered by an adequate window in the frequency domain. And then, $C(f, y)$ can be obtained by spectrum shift center. Taking inverse Fourier transform of $C(f, y)$, we can get $c(x, y)$. The phase change of the deformed pattern is

$$
\begin{aligned}
\Phi(x, y) & =\arctan \left\{\frac{\operatorname{Im}[c(x, y)]}{\operatorname{Re}[c(x, y)]}\right\}+2 n \pi \\
& =\arctan \frac{G}{F}+2 n \pi=\Phi^{\prime}(x, y)+2 \pi n
\end{aligned}
$$

where $F=\operatorname{Re}[c(x, y)]$ and $G=\operatorname{Im}[c(x, y)]$ represent the real and imaginary parts of $c(x, y)$, respectively. $\Phi^{\prime}(x, y)$ is the principal phase which is in the range of $[-\pi, \pi]$. A phase unwrapping procedure should be taken to get the continuous actual phase distribution $\Phi(x, y)$.

The purpose of the spectrum shift center procedure is to remove the linear term in the phase. However, because of the limitation of the image resolution, there will be a residual linear term error. Fortunately, the fringe pattern image of the reference plane can be used to obtain the linear term of phase in advance. After subtracting the reference phase, the real phase can be obtained by the Fourier transform without spectrum shift.

\section{The Experimental System}

The schematic diagram of the experimental system is shown in Figure 2. In the measurement research of this paper, the experimental system is contained with two parts, the first one is the impact setup, and the second one is the measurement part. In the impact setup, the metallic components were launched by a gas gun or a spin fan machine so that it can be pull out with a high initial speed. The impact speed when the metallic components arrived at the reference surface can be controlled by the parameters of the gas gun or the velocity of the spin fan. Thus we can obtain different experimental results about the plate deformation. It is helpful for us to investigate the relationship between the impact energy and the surface deformation, thus we can redesign the strength of the detected plate which was made by the composite materials. 


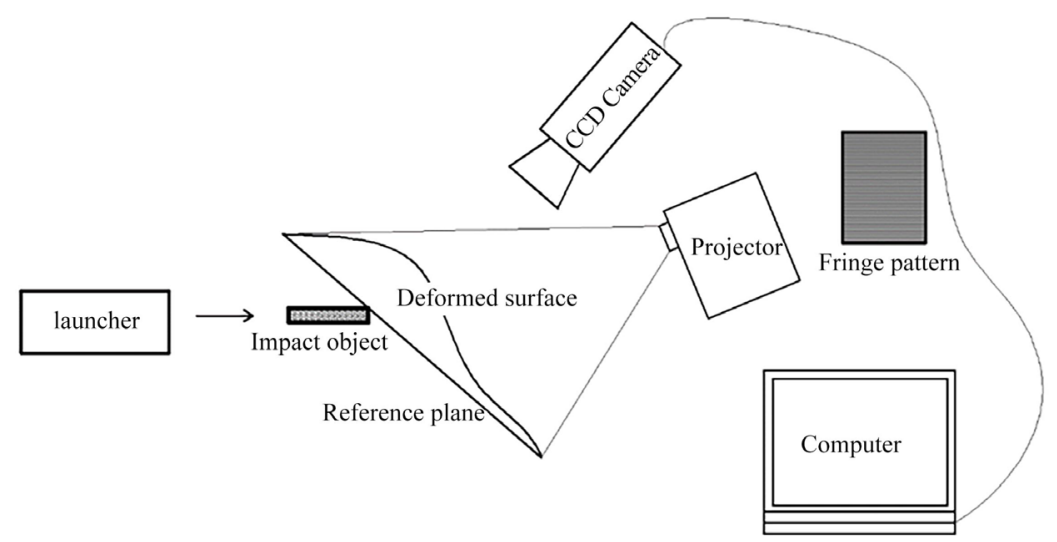

Figure 2. Sketch map of the experimental setup.

In order to obtain the surface deformation of the composite plates, the proposed projection grating profilometry was used with the measurement part. The deformation measurement experimental setup is constructed by a high-speed CCD camera, a digital projector, and a personal computer. The camera manufactured by NAC Image Technology Inc. has a very low noise rating, and it also boasts the highest dynamic range to $64 \mathrm{~dB}$. It has high speed and high resolution $(800 \times 528,60,000 \mathrm{fps})$, which is suitable for the impact procedure measurement research. By using the digital projector, only one frame of parallel fringe pattern with cosine function modulated intensity is projected onto the detected object with an incidence angle. The classical fringe patterns are shown in Figure 3, and the pitch of the fringe pattern can be different with different deformation measurement accuracy requirements.

The high-speed CCD camera is fixed normal to the reference plane, and the distance between the camera and the detected object depends on the size of the composite plate and the parameter. When the impact object was launched, the camera would be triggered by input signal. So that the deformed fringe patterns modulated by the surface profile of the detected object can be captured in the impact procedure. Here, the most important thing is how to keep the impaction and the capturing in Synchronize. And then, the deformed fringe pattern images are recorded in the personal computer for the next calculation. After a measurement system calibration test, the surface profile can be extracted by calculating the fringe deformation between the reference and the detected surface.

\section{Calibration Test Method}

In order to obtain the coefficient $k$ defined in Equation (4), the calibration test should be carried out before the impact test. A specimen with a special shape, which is shown in Figure 4, is manufactured by a 3D printer. The calibration specimen should be posted onto the reference plane to calibrate the optical projection system. The fringe pattern images of corresponding to the reference plane and the calibration specimen surface are shown in Figure 5, respectively. By using the previous Fourier transform algorithm the phase deformation of the two fringe patterns is extracted and shown in Figure 6. 


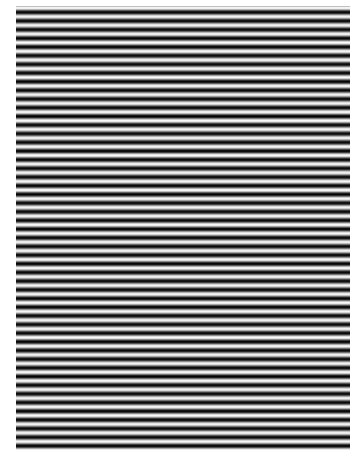

(a)

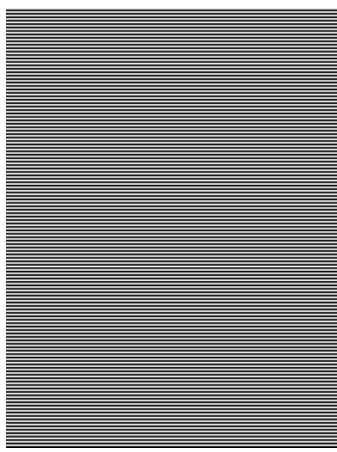

(b)

Figure 3. Projected fringe patterns (a) pitch $=20$ pixels (b) pitch $=8$ pixels.
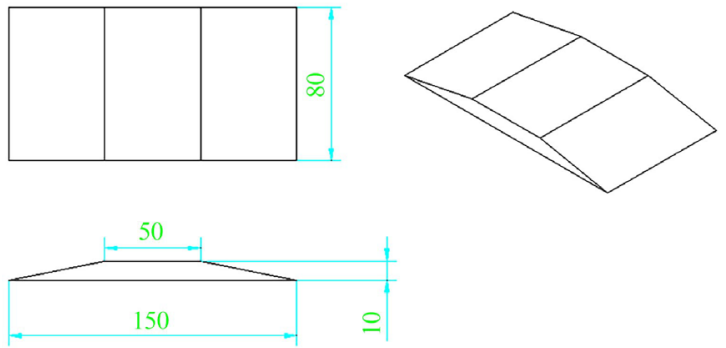

Figure 4. Calibration specimen.

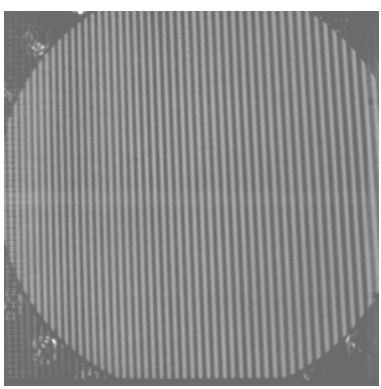

(a)

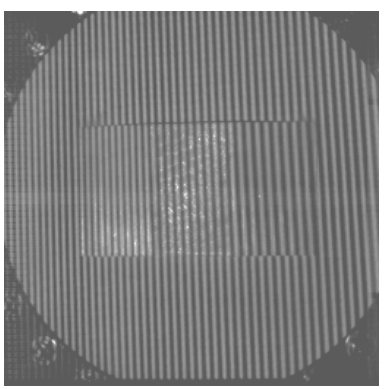

(b)

Figure 5. Calibration fringe patterns (a) reference (b) specimen.

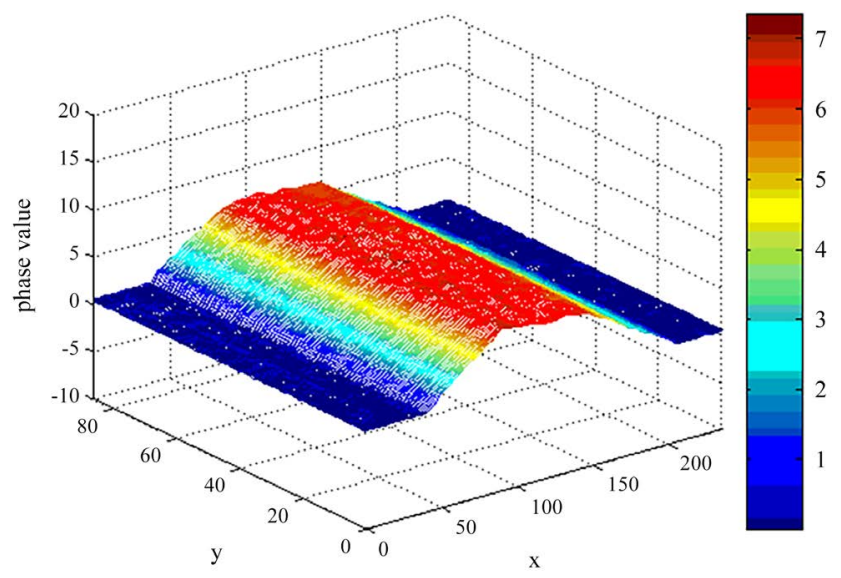

Figure 6. Extracted profile of the calibration specimen. 
In order to improve the calculation accuracy of the calibration test, some different calibration specimens were designed to investigate the calibration error. The maximum height of the $3 \mathrm{D}$ printed specimen is $10,20,30,40,50,60 \mathrm{~mm}$, respectively. Calibration can be obtained through the calculation of arithmetic average specimen surface height of the maximum value. The calculated value of the phase difference with the different height is shown in Figure 7(a), and the error distribution is shown in Figure 7(b). From the calibration experimental results, it is found that the calibration error is about $5-7$ percent, which can be acceptable for the measurement in the impact deformation. However, the calibration error is growing with the maximum height of the calibration specimen, so that the measurement error will also be bigger corresponding to the surface deformation value which caused by the huge impact energy.

\section{Experimental Result and Analysis}

\subsection{Gas Gun Projectile Testing}

Firstly, the manufactured composite plates are tested by a gas gun impact testing system. The compressed gas was used as the propellant to launch the impact object. In order to simulate different impaction situation, the angle between the projectile and the target can be changed during the testing. The size of the manufactured composite plate was approximately $180 \mathrm{~mm} \times 400 \mathrm{~mm}$, and the plate was fixed to a rigid testing fixture. The fringe pattern was projected onto the target, and then high speed camera was used to record the images during the impaction procedure. In this test, the time interval of the high speed camera was set as $27 \mu$ s. Totally, there are 150 frames of image are captured and restored. The out-of-plane deformation can be extracted by using the projection grating profilometry method.

One of the composite plate impact test results are shown as follow. Figure 8 is the captured fringe patterns; it is obviously that there is a difference between the reference plane and the deformed composite plate plane. The fringe distortion could be found in Figure $8(\mathrm{~b})$, and the surface deformation information

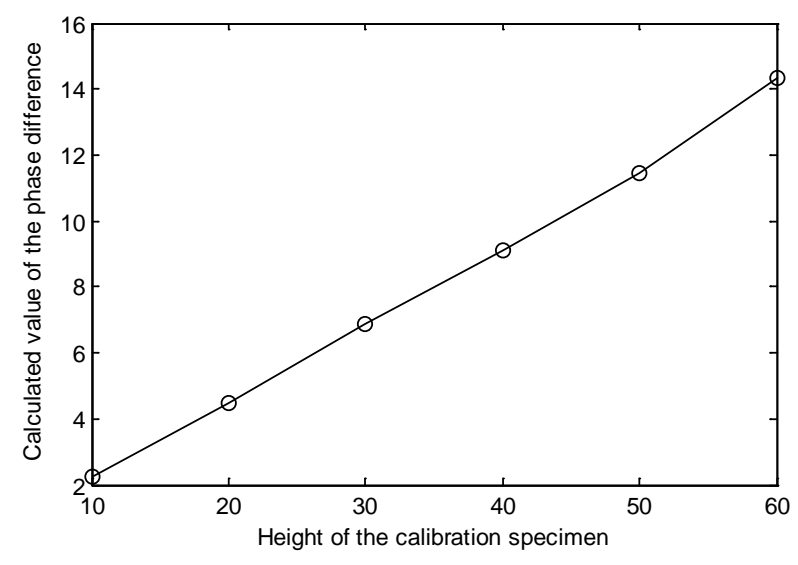

(a)

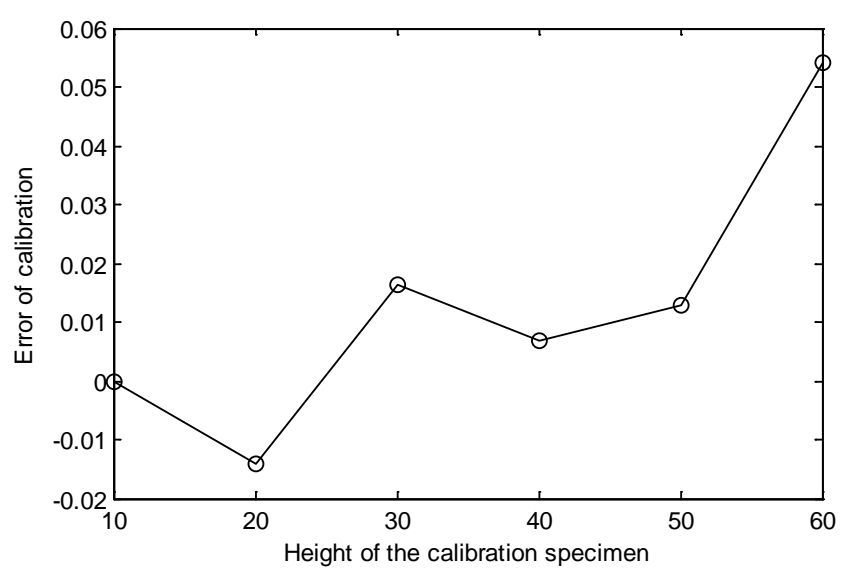

(b)

Figure 7. Calibration error analysis (a) phases difference distribution (b) error distribution. 


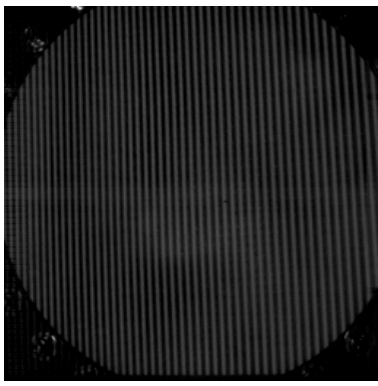

(a)

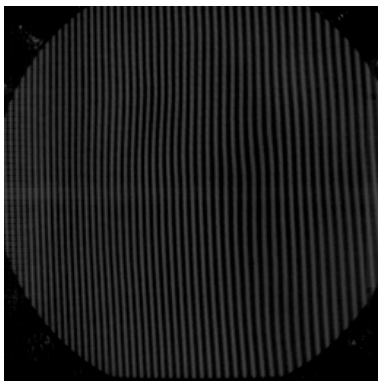

(b)

Figure 8. Captured fringe patterns of composite plate (a) reference (b) deformed.

was contained in that distortion. By using Fourier transform algorithm, the extracted phase distribution without unwrapping is shown in Figure 9. And then, the real phase distribution can be calculated with a phase unwrapping processing, so that the phase difference between the reference plane and the detected composite plate can be obtained automatically. As we know, the coefficient of the measurement system can be calibrated by the proposed calibration experiment. In this test the calibration test result is $k=1.5747 \mathrm{~mm} / \mathrm{rad}$. Finally, the experimental results of the surface deformation are shown in Figure 10.

\subsection{Spin Fan Projectile Testing}

In this part, the measurement method was used to obtain the deformation of a fan case which was also manufactured by the same composite material as previous test. The impaction experiment was simulated by a spin fan testing, and the projectile was launched by a motor at a high speed up to $400 \mathrm{~m} / \mathrm{s}$. In this research, we have two different tests to study the different impact energy influence of the fan case strength. The experimental results are shown as follow. Comparing the captured fringe patterns of composite plate which were shown in Figure 11 and Figure 12, the impact energy of the test A is bigger than that of the test $\mathrm{B}$. Thus the fan case was totally broken in the impaction procedure. It is obviously shown in Figure 11, and there is a big difference between the reference and the deformed case because of the fringe distortion. By using the same image process and phase extracting algorithm, we can get the phase difference distribution. And then, the coefficient of the measurement system can be calibrated by the proposed calibration experiment. In the test- $\mathrm{A}$ and test- $\mathrm{B}$ the calibrated coefficient values are $K=10.0125 \mathrm{~mm} / \mathrm{rad}$ and $K=6.8173 \mathrm{~mm} / \mathrm{rad}$, respectively. Finally, the experimental results of the surface deformation are shown in Figure 13 and Figure 14, respectively.

In order to study the characteristics of the fan case surface deformation in the whole impaction procedure, five points were selected to compare the deformation in different area and different period of the case. The compared results are shown in Figure 15 and Figure 16, respectively. Firstly, the values of the surface deformation are different corresponding to the different measurement positions on the fan case surface. The maximum deformation position is the impaction 
point of the projectile, and the deformation of the other points is becoming smaller than the impaction point due to the decreasing of the impact energy concentration. However, the trend of deformation variety of each measurement point is the same. Comparing the two different experimental results in Figure 15 and Figure 16, it is also obviously that the characteristics of the fan case surface deformation are also different. Because the impact energy is big enough, the fan case was broken in the test-A. The surface deformation is suddenly bigger than before about at 0.7 second in the whole impaction procedure. After that broken, the surface deformation was fall back. However, the surface deformation

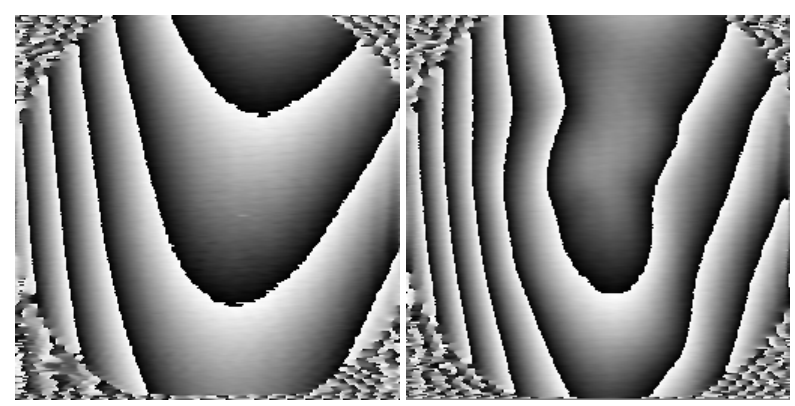

(a)

(b)

Figure 9. Extracted phase distribution without unwrapping (a) reference (b) deformed.

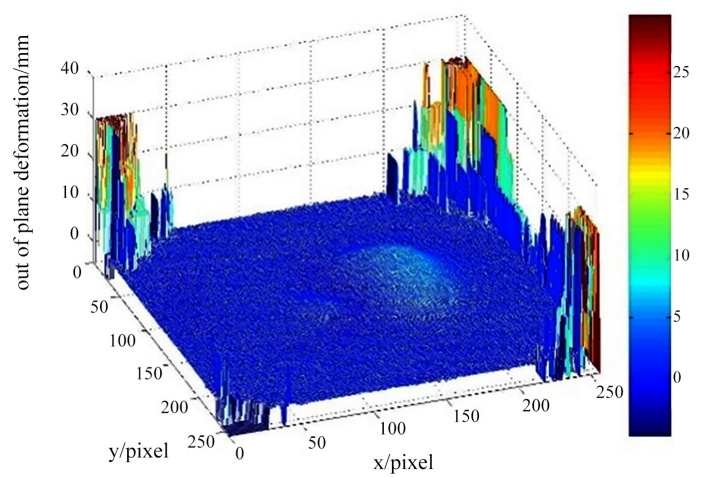

(a)

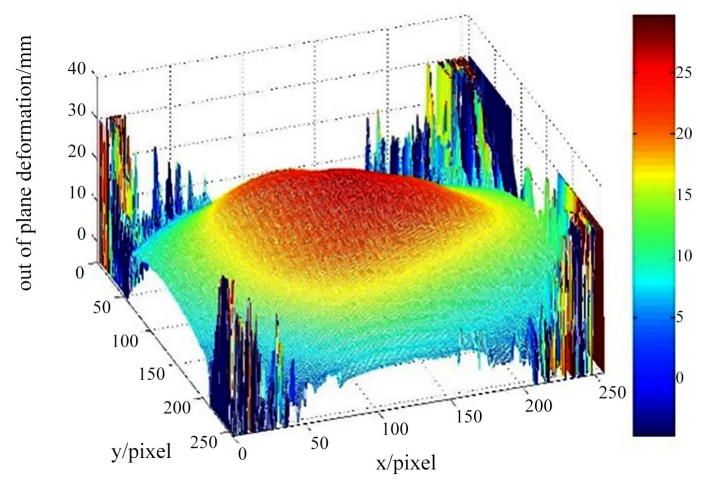

(c)

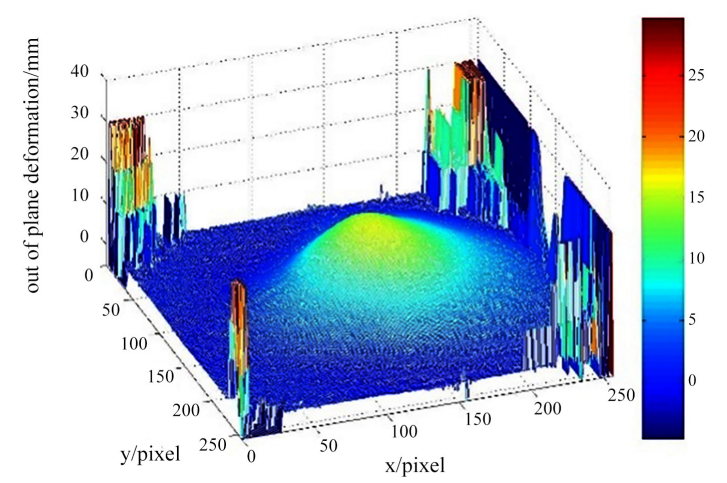

(b)

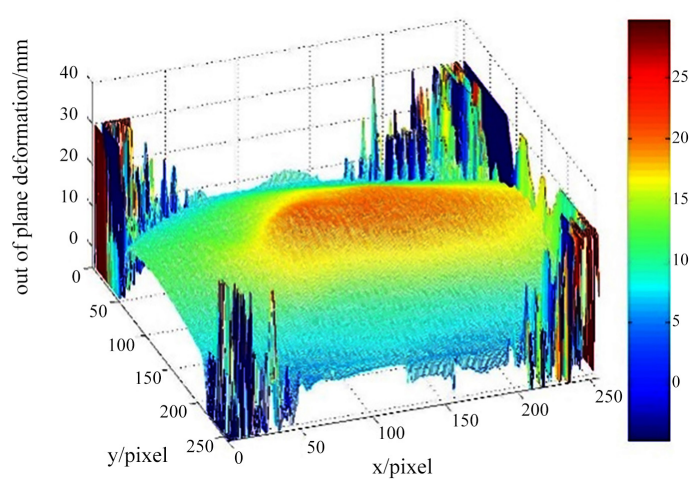

(d)

Figure 10. Extracted profile of the tested composite material plate. 


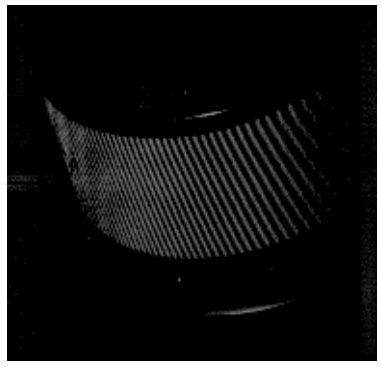

(a)

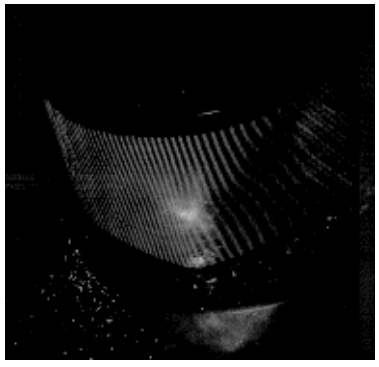

(b)

Figure 11. Captured fringe patterns of composite plate-Test A (a) reference (b) deformed.

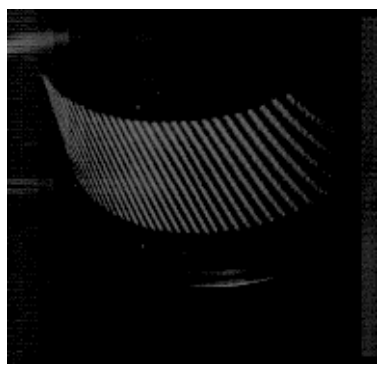

(a)

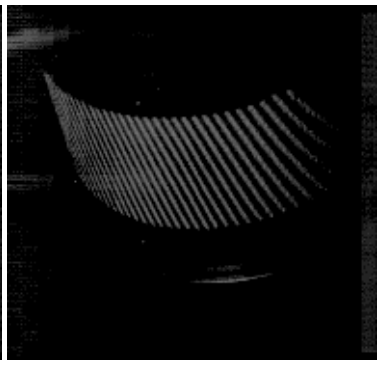

(b)

Figure 12. Captured fringe patterns of composite plate-Test B (a) reference (b) deformed.

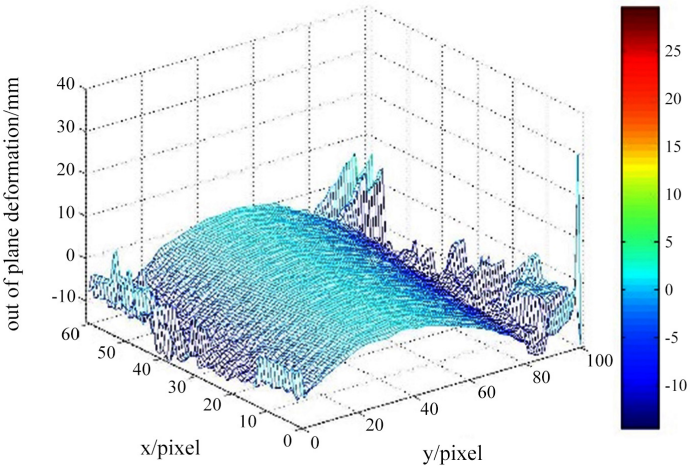

(a) 1

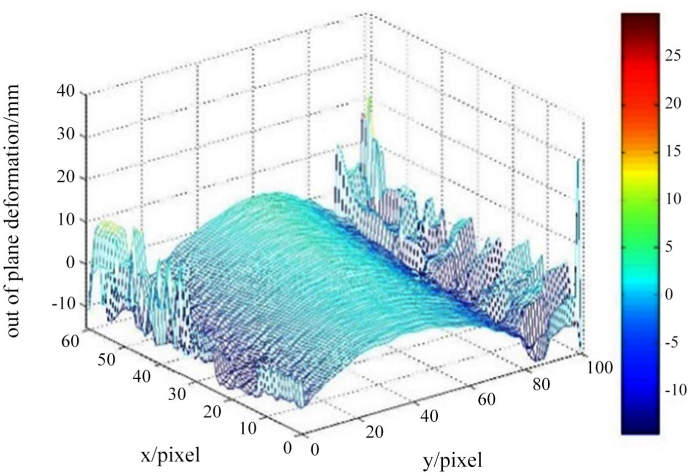

(c) 301

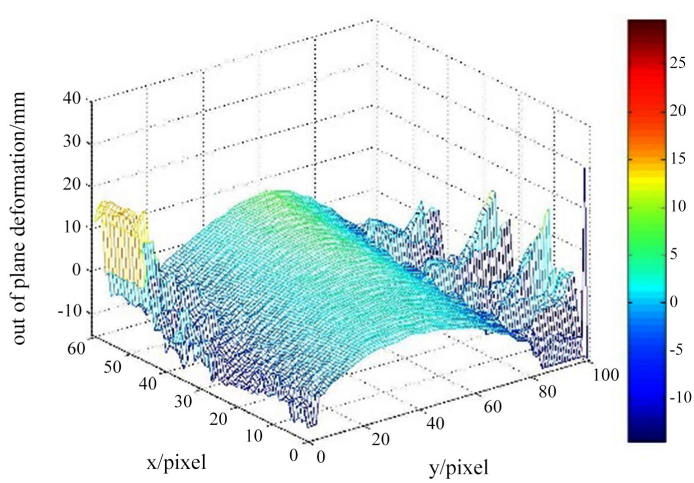

(b) 101

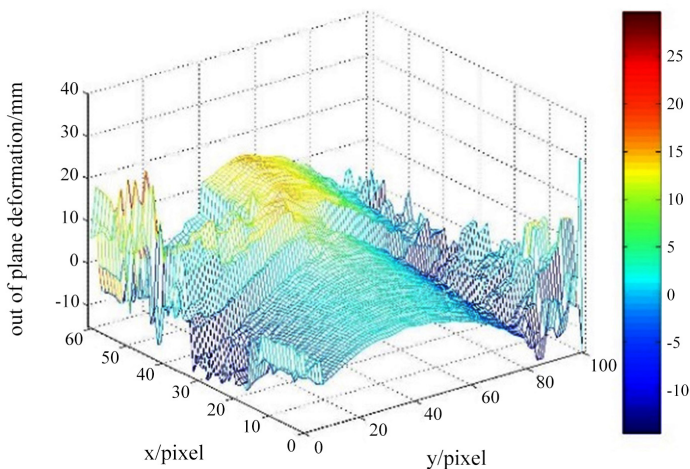

(d) 481

Figure 13. Extracted profile of the tested fan case-Test A. 


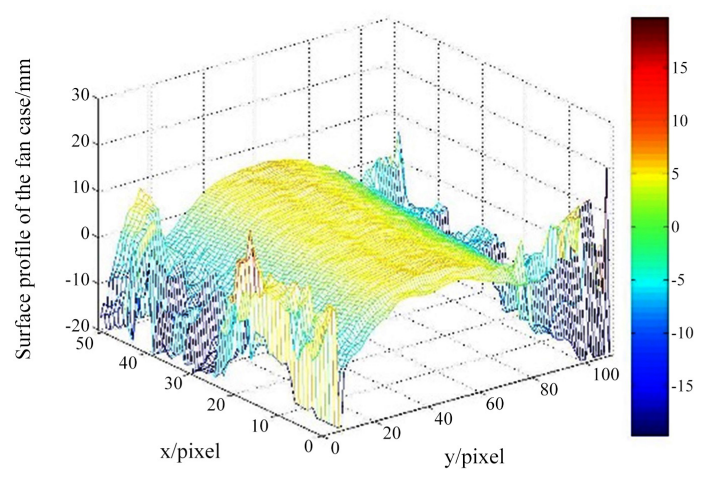

(a) 10

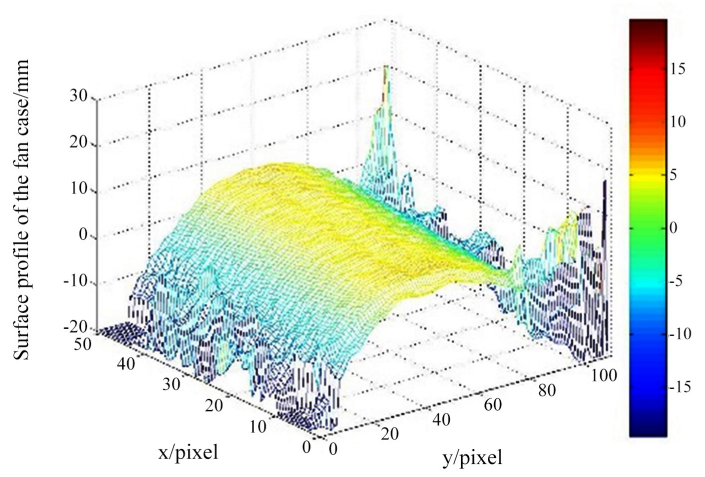

(c) 301

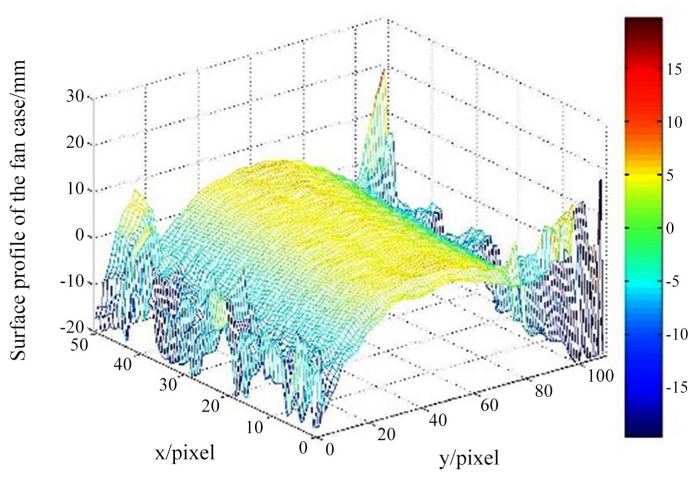

(b) 110

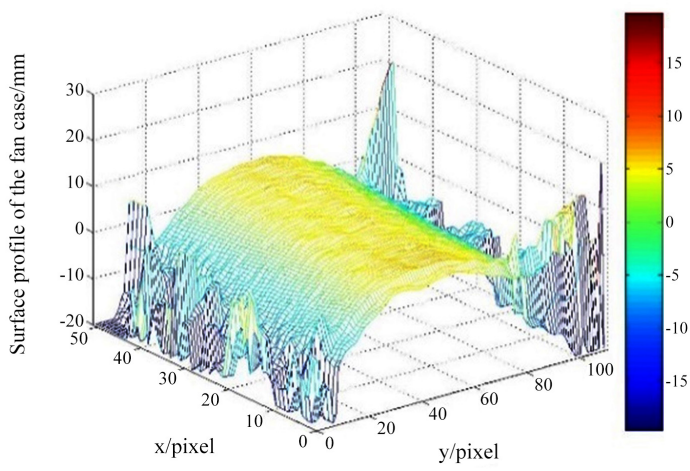

(d) 481

Figure 14. Extracted profile of the tested fan case-Test B.

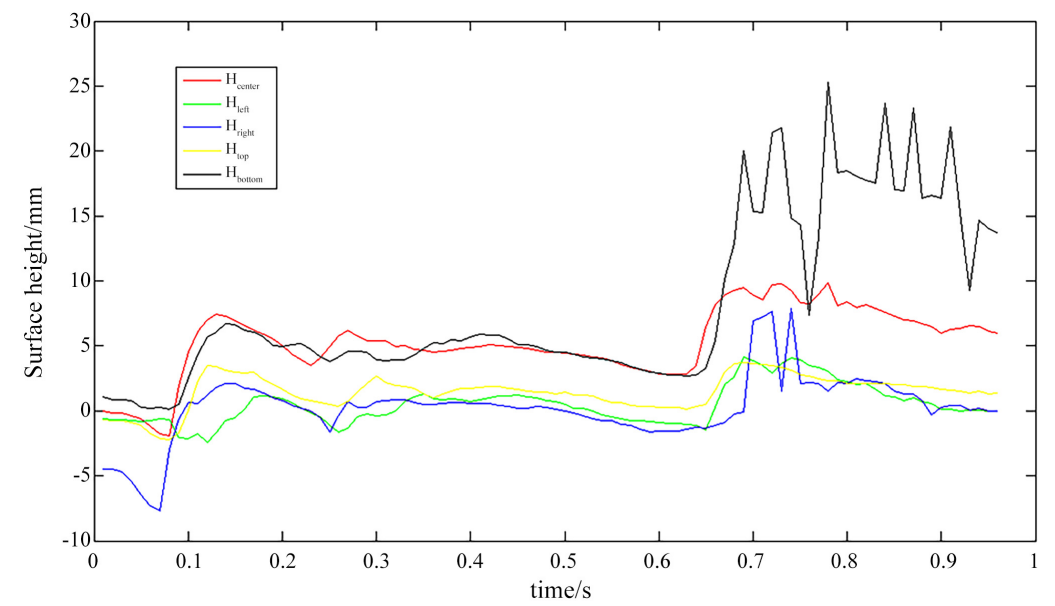

Figure 15. Surface deformation values distribution of the tested fan case-Test A.

of the fan case in test-B present different characteristics. Because the impaction energy is much smaller than that in the test- $\mathrm{A}$, so the fan case was keeping completely in the whole impaction procedure. From the experimental results shown in Figure 16, there is a periodic fluctuation in the deformation curves during the time axis; it looks like that the impaction inducing a surface vibration of the detected fan case. It is meaning that the fan case surface can keep well, when resist the strength is enough to the lower energy impaction. 


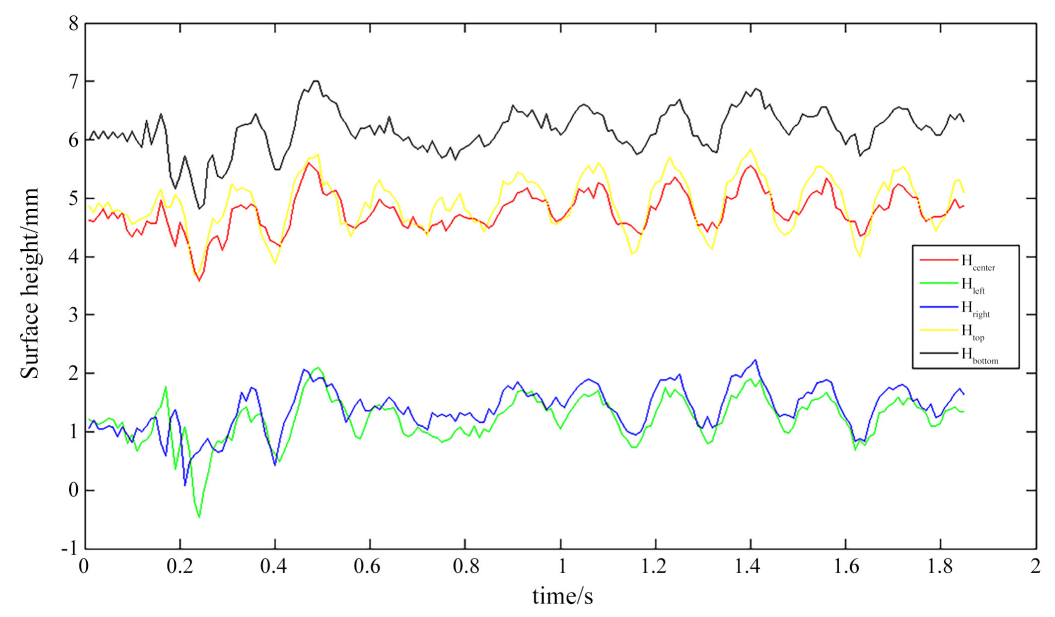

Figure 16. Surface deformation values distribution of the tested fan case-Test B.

\section{Conclusion}

The surface impaction deformation can be measured by the projection profilometry method. An experimental system was combined by the impaction and the deformation inspection parts. By using the high speed image capturing and recording system, the Fourier transform is successfully applied for the phase difference extracting. From the experimental results, it is found that the calibration error is about 5 - 7 percent, which is acceptable for the measurement in the impact deformation. The detected composite plate and circle fan case were used to prove the feasibility of the proposed method.

\section{Acknowledgements}

The author gratefully acknowledges support from the National Natural Science Foundation of China (Grant No. 51875568).

\section{Conflicts of Interest}

The authors declare no conflicts of interest regarding the publication of this paper.

\section{References}

[1] Hu, E.Y. and He, Y.M. (2009) Surface Profile Measurement of Moving Objects by Using an Improved $\pi$ Phase-Shifting Fourier Transform Profilometry. Optics and Lasers in Engineering, 47, 57-61. https://doi.org/10.1016/j.optlaseng.2008.08.003

[2] Geng, Z.J. (1996) Rainbow 3D Camera: New Concept of High-Speed Three Vision System. Optical Engineering, 35, 376-383. https://doi.org/10.1117/1.601023

[3] Feng, S.J., Chen, Q., Zuo, C., Sun, J.S. and Yu, S.L. (2014) High-Speed Real-Time 3-D Coordinates Measurement Based on Fringe Projection Profilometry Considering Camera Lens Distortion. Optics Communications, 329, 44-56. https://doi.org/10.1016/j.optcom.2014.04.067

[4] Wang, Y., Gupta, M., Zhang, S., Wang, S., Gu, X.F., Samaras, D. and Huang, P.S. (2008) High Resolution Tracking of Non-Rigid 3D Motion of Densely Sampled Data Using Harmonic Maps. International Journal of Computer Vision, 76, 283-300. https://doi.org/10.1007/s11263-007-0063-y 
[5] Towers, C.E., Towers, D.P. and Jones, J.D.C. (2005) Absolute Fringe Order Calculation Using Optimized Multi-Frequency Selection in Full-Field Profilometry. Optics and Lasers in Engineering, 43, 788-800.

https://doi.org/10.1016/j.optlaseng.2004.08.005

[6] Rae, P.J., Goldrein, H.T., Bourne, N.K., et al. (1999) Measurement of Dynamic Large-Strain Deformation Maps Using an Automated Fine Grid Technique. Optics and Lasers in Engineering, 31, 113-122. https://doi.org/10.1016/S0143-8166(99)00003-2

[7] Sevenhuijsen, P.J., Sirkis, J.S. and Bremand, F. (1993) Current Trends in Obtaining Deformation Data from Grids. Experimental Techniques, 17, 22-26. https://doi.org/10.1111/j.1747-1567.1993.tb00747.x

[8] Malacara, D., Servín, M. and Malacara, Z. (2005) Interferogram Analysis for Optical Testing. CRC Press, Boca Raton. https://doi.org/10.1201/9781420027273

[9] Chen, Y.M., He, Y.M. and Hu, E.Y. (2008) Phase Deviation Analysis and Phase Retrieval for Small Intensity Saturation in Phase-Shifting Projected Fringe Profilometry. Optics Communications, 281, 3087-3090. https://doi.org/10.1016/j.optcom.2008.01.070

[10] Takeda, M. and Mutoh, K. (1983) Fourier Transform Profilometry for the Automatic Measurement of 3-D Object Shapes. Applied Optics, 22, 3977-3982. https://doi.org/10.1364/AO.22.003977

[11] Takeda, M. (1990) Spatial-Carrier Fringe Pattern Analysis and Its Application to Precision Interferometry and Profilometry: An Overview. Industrial Metrology, 1, 79-99. https://doi.org/10.1016/0921-5956(90)80019-R

[12] He, Y.M., Tay, C.J. and Shang, H.M. (1998) Deformation and Profile Measurement Using the Digital Projection Grating Method. Optics and Lasers in Engineering, 30, 367-377. https://doi.org/10.1016/S0143-8166(98)00040-2

[13] Su, X.Y. and Chen, W.J. (2001) Fourier Transform Profilometry: A Review. Optics and Lasers in Engineering, 35, 263-284.

https://doi.org/10.1016/S0143-8166(01)00023-9

[14] Su, X.Y. and Zhang, Q.C. (2010) Dynamic 3-D Shape Measurement Method: A review. Optics and Lasers in Engineering, 48, 191-204. https://doi.org/10.1016/j.optlaseng.2009.03.012

[15] Zhang, S. and Huang, P. (2004) High-Resolution, Real-Time 3-D Shape Acquisition. 2004 IEEE Computer Vision and Pattern Recognition Workshop, Washington DC, 27 June-2 July 2004, 28. https://doi.org/10.1109/CVPR.2004.360

[16] Huang, P.S., Zhang, C. and Chiang, F.P. (2003) High-Speed 3-D Shape Measurement Based on Digital Fringe Projection. Optical Engineering, 42, 163-168. https://doi.org/10.1117/1.1525272 